\title{
nomograma para determinar la tensión de
}

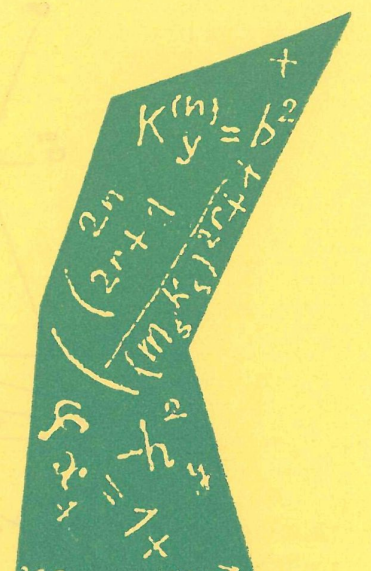

R. MUÑOZ MARTIALAY

S I N O P S I S

Nomograma de alineación para determinar, rápidamente, la tensión de fisuración en laø piezas de hormigón armado con redondos lisos o barras corrugadas.

En el número 89 de esta revista se publicó un artículo titulado: «Una fórmula para determinar la tensión de fisuración».

En este trabajo se propone una fórmula para determinar la referida tensión en función del diámetro de las armaduras, de su cuantía, luz admitida para la grieta y recubrimiento en las piezas de hormigón armado, admitiendo una resistencia de $13 \mathrm{~kg} / \mathrm{cm}^{2}$ para el hormigón trabajando a tracción.

Como las operaciones numéricas de dicha fórmula son laboriosas, se ha hecho un nomograma de alineación que acompaña a este t con objeto de facilitar dichas operaciones.

La expresión original es la siguiente:

$$
\sigma_{f}=\frac{13}{q+0,01}+34.000 \sqrt{\frac{\mathrm{Ag} \cdot \mathrm{K}}{\left(1+\frac{\varnothing}{\delta_{1}}\right)\left(1+\frac{\varnothing}{2}\right)(1+\varnothing)}}
$$

siendo: $\sigma_{f}$, la tensión de fisuración, en kilogramos por centímetro cuadrado $q$, la cuantía o relación entre las secciones del acero y del hormigón

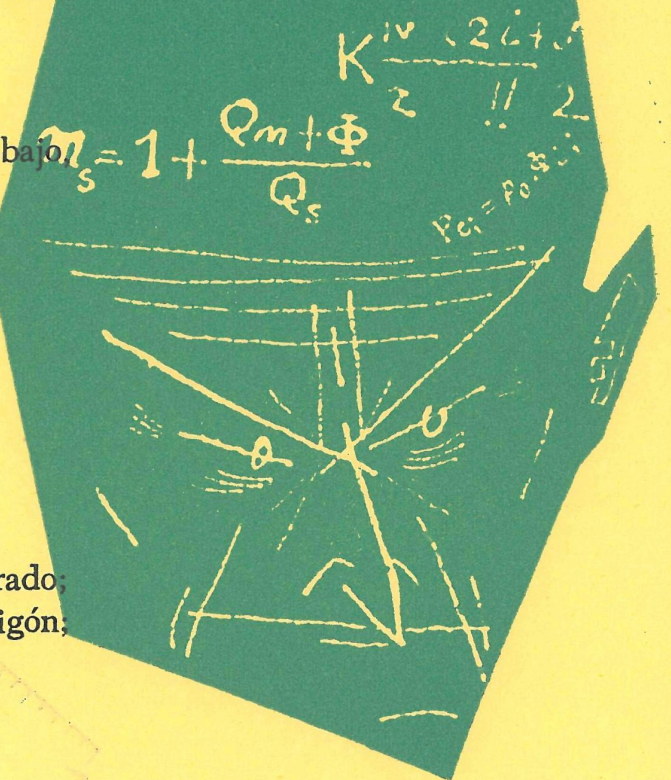




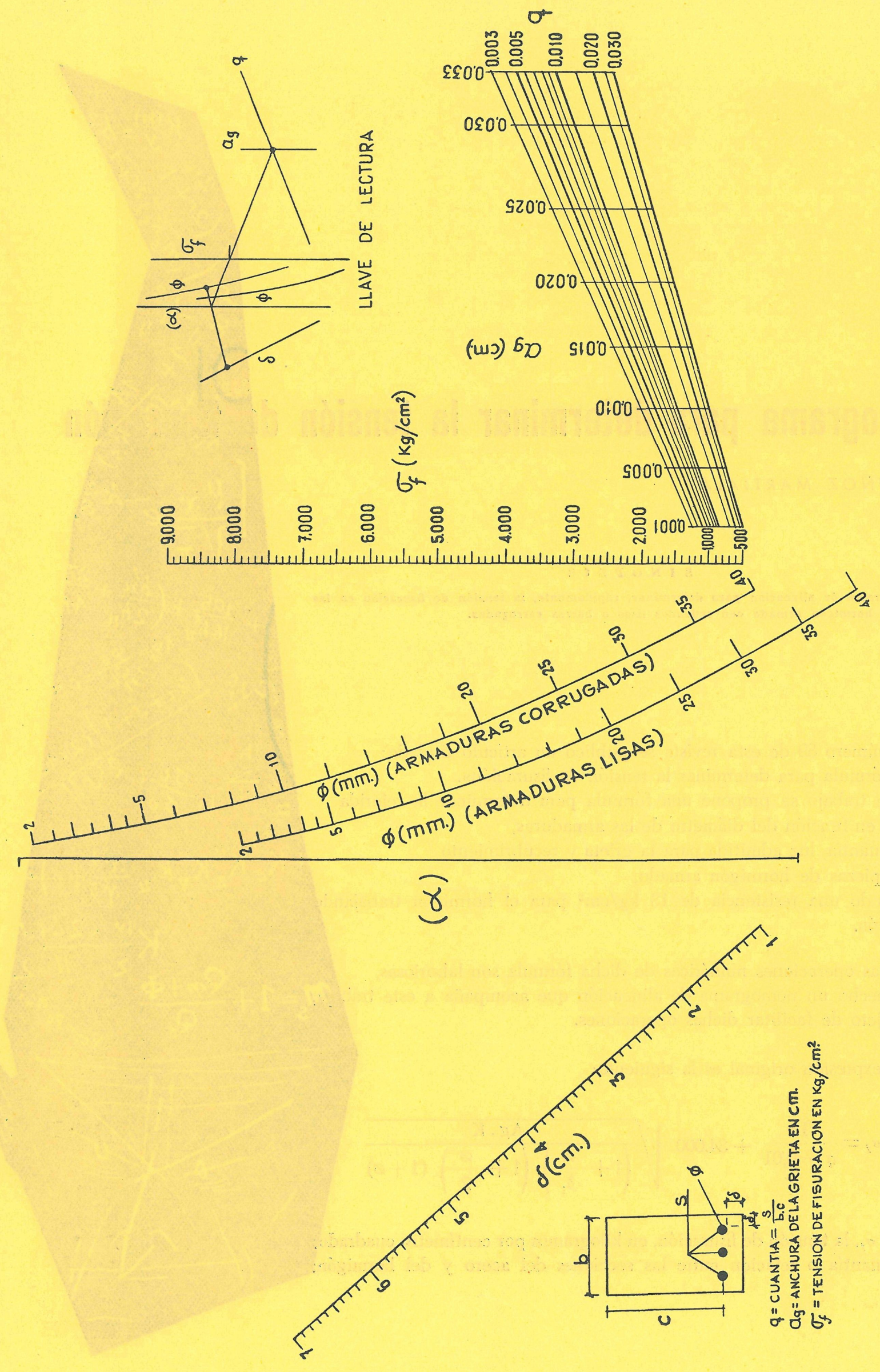


Ag, la luz admisible de la grieta, en centímetros; $\varnothing$, el diámetro del redondo, en centímetros; $\delta_{1}$ y $\delta_{2}$, los recubrimientos o distancias de la superficie de la barra a los paramentos de cabeza y canto de la viga, respectivamente, y K es una constante de valor 1 para armaduras lisas y 1,8 en las corrugadas.

Es práctica corriente en la construcción la admisión de un recubrimiento único para todas las armaduras, en cuyo caso $\delta_{1}=\delta_{2}=\delta$, por lo que la expresión anterior toma la forma siguiente:

$$
\sigma_{f}=\frac{13}{q+0,01}+\frac{34.000}{1+\frac{\varnothing}{\delta}} \sqrt{\frac{\mathrm{Ag} \cdot \mathrm{K}}{1+\varnothing}}
$$

Partiendo de esta expresión se ha dibujado el nomograma adjunto, empleando las mismas unidades para el recubrimiento, cuantía y luz de la grieta. Los diámetros de los redondos se expresan en milímetros, ya que corrientemente se les denomina así.

manejo del nomograma

Se empieza llevando el valor $\delta$ a la escala de recubrimientos y el de $\varnothing$ en la correspondiente a los diámetros, teniendo cuidado de utilizar la que se refiere a la armadura que nos interesa, es decir, tipo liso o corrugado. La recta que une estos dos puntos cortará a la charnela $(\alpha)$ en un tercer punto, que, unido al de la intersección de las rectas correspondientes a los valores de $\mathrm{Ag}$ y $q$, cortará a la escala $\sigma_{f}$ en un cuarto punto, que, finalmente, nos dará el valor de la tensión de fisuración.

Inversamente, se puede suponer conocida la tensión de fisuración y tres de las cuatro variables restantes, en cuyo caso el nomograma nos dará la quinta.

Efectivamente, tratemos de hallar la luz admisible de la grieta en función de la tensión, recubrimiento, diámetro de la armadura y cuantía; se procederá como se dijo anteriormente para determinar el punto de la charnela, que, unido con el correspondiente a la tensión, definen una recta que cortará a la de la cuantía en un punto tal, que por interpolación entre las rectas de la luz de grieta nos dará la luz de ésta. Siguiendo un procedimiento similar se pueden obtener otros valores en función de los restantes.

ejemplo númerico de aplicación del nomograma

El manejo del nomograma no ofrece dificultad alguna; en efecto: supongamos una viga en la cual $\delta=2 \mathrm{~cm} ; \phi=16 \mathrm{~mm}$ (armadura lisa); $\mathrm{Ag}=0,020 \mathrm{~cm}$, y $q=0,01$. La tensión de fisuración se obtiene de la forma siguiente:

Se empieza uniendo el punto 2 de la escala $\delta$ con el 16 de la escala $\varnothing$ (armadura lisa). Después se une la de intersección de esta recta con la charnela $(\alpha)$ con la intersección de las líneas $\mathrm{Ag}=0,020$ y $q=0,01$. Esta última recta cortará la escala $\sigma_{f}$ en un punto cuyo valor, en este caso 2.300 , representa la tensión de fisuración en kilogramos por centímetro cuadrado.

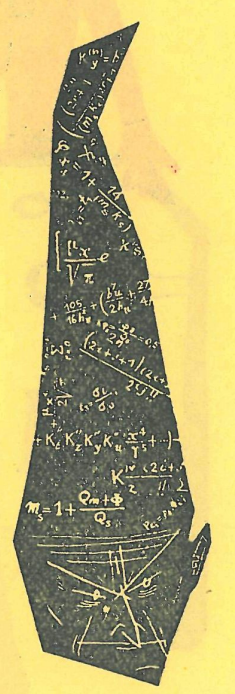

